\title{
Some remarks concerning the thermodynamics of the simple ideal gas and related mathematical background
}

\author{
Győző G. Láng ${ }^{1}$
}

Received: 1 March 2016/Accepted: 5 May 2016/Published online: 6 June 2016

(C) Springer International Publishing Switzerland 2016

\begin{abstract}
This study is intended to give a brief overview of some of the mathematical concepts and techniques relevant to the study of thermodynamics, including the theory of homogeneous functions and partly homogeneous functions, Euler's theorem, and Legendre transformation. The basic thermodynamic functions of an ideal monatomic gas (fundamental relations and thermodynamic potential functions) have been selected as illustrative examples.
\end{abstract}

Keywords Ideal gas · Fundamental relation ·

Homogeneous functions - Partly homogeneous functions . Euler's theorem · Legendre transformation

\section{Introduction}

An ideal gas is a theoretical gas composed of many randomly moving point particles. The essence of ideal gas behavior is that the molecules of the gas do not interact except when they collide elastically. A substance is said to be an ideal gas $[1,2,3]$.

(a) if it satisfies the following equation (mechanical equation of state):

$p V=n R T=\frac{m}{M} R T$

Győző G. Láng

langgyg@chem.elte.hu

1 Institute of Chemistry, Laboratory of Electrochemistry and Electroanalytical Chemistry and Department of Physical Chemistry, Eötvös Loránd University, Budapest Pázmány P. s. 1/A, Budapest 1117, Hungary where $n$ is the (chemical) amount, $m$ is the mass, $M$ is the molar mass of the substance, $p$ is the pressure, $V$ is the volume, $T$ is the temperature, and $R$ is the "universal" gas constant, respectively, (b) for a single-component ideal gas the molar internal energy $(u)$ is a function only of the temperature (and inversely), i.e.,

$u=u(T)$

(c) and the Helmholtz potential (or Helmholtz free energy) $F\left(T, V, n_{1}, n_{2}, \ldots, n_{m}\right)$ of a multicomponent ideal gas is additive over the components:

$F\left(T, V, n_{1}, n_{2}, \ldots, n_{m}\right)=F\left(T, V, n_{1}\right)+F\left(T, V, n_{2}\right)+\cdots$ $+F\left(T, V, n_{m}\right)$

(The latter relationship is sometimes referred to as "Gibbs's theorem").

In the IUPAC Gold Book [4] the ideal gas is defined as follows:

"Ideal gas. Gas which obeys the equation of state $p V=n R T$ (the ideal gas law; $p$ is the pressure, $V$ the volume, $n$ the amount of molecules, $R$ the gas constant and $T$ the thermodynamic temperature). For an ideal gas the fugacity, $f$, of each constituent $\mathbf{B}$ is equal to its partial pressure, $p_{\mathrm{B}}, f_{\mathrm{B}}=p_{\mathrm{B}}=x_{\mathrm{B}} \cdot p$, where $x_{\mathrm{B}}$ is the amount fraction of $\mathbf{B}$ ".

In many textbooks the ideal gas is defined as a gas whose particles don't have any volume of their own and as a gas where no interactions between the particles occur (that is, there are no intermolecular potential energies). On the other hand each of these particles has a mass, that is, the molecules are point particles.

A "simple ideal gas" is characterized by the two equations $p V=n R T$ and $U=c n R T$ where $c$ is a constant and $U$ is the internal energy [1]. 


\section{Fundamental relations for the ideal gas}

The compact form of the entropy fundamental relation (fundamental equation, fundamental function) of a "simple" ideal gas can be given as [see Ref. [1], p. 68, Eq. 3.34, or "Appendix 1", Eq. (59)].

$S=n s_{0}+n R \ln \left[\left(\frac{U}{U_{0}}\right)^{c}\left(\frac{V}{V_{0}}\right)\left(\frac{n}{n_{0}}\right)^{-(c+1)}\right]$

In Eq. (1) $S$ is the entropy, $U$ is the internal energy, $V$ is the volume of the system, $n$ is the chemical amount of substance of the gas (1 component), respectively, $c$ is a constant ("monatomic ideal gases" have a value of $c=3 / 2$ ), $R$ is the "universal gas constant", $U_{0}, n_{0}$ and $V_{0}$ are the parameters of a fixed reference state, and

$s_{0}=\left(S_{0} / n_{0}\right)=(c+1) R-(\mu / T)_{0}$.

In Eq. (2) $(\mu / T)_{0}$ is an undetermined integration constant (see "Appendix 1"). If the integration constant is known, then Eq. (1) contains all possible thermodynamic information about a simple ideal gas. Thus, for the special case of an ideal monatomic gas we can write:

$S(U, V, n)=\frac{n}{n_{0}} S_{0}+n R \ln \left[\left(\frac{U}{U_{0}}\right)^{\frac{3}{2}}\left(\frac{V}{V_{0}}\right)\left(\frac{n}{n_{0}}\right)^{-\frac{5}{2}}\right]$

or

$$
\begin{aligned}
U(S, V, n) & =U_{0}\left(\frac{n}{n_{0}}\right)^{\frac{5}{3}}\left(\frac{V_{0}}{V}\right)^{\frac{2}{3}} \exp \left[\frac{2}{3 R}\left(\frac{S}{n}-\frac{S_{0}}{n_{0}}\right)\right] \\
& =n U_{0}\left(\frac{n}{V}\right)^{\frac{2}{3}} \frac{V_{0}^{2 / 3}}{n_{0}^{5 / 3}} \exp \left[\frac{2}{3 R}\left(\frac{S}{n}-\frac{S_{0}}{n_{0}}\right)\right] .
\end{aligned}
$$

The function $U(S, V, n)$ given is Eq. (4) can be called the fundamental function of the internal energy of the ideal gas with the entropy $S$, the chemical amount $n$, and the volume $V$ as the respective fundamental variables. This function is probably the simplest example of a fundamental function (fundamental relation) in thermodynamics which can be given in an explicit form. On the other hand, this function offers the opportunity to study and demonstrate the application of important mathematical concepts (e.g., the theory of homogeneous functions and partly homogeneous functions, Euler's theorem, and the Legendre transformation $[5,6])$ to real thermodynamic problems.

For instance multiplying all independent variables of the function $U$ simultaneously by the same factor $k>0$ we obtain:

$$
\begin{aligned}
U(k S, k V, k n)= & k n U_{0}\left(\frac{k n}{k V}\right)^{\frac{2}{3}} \frac{V_{0}^{2 / 3}}{n_{0}^{5 / 3}} \\
& \times \exp \left[\frac{2}{3 R}\left(\frac{k S}{k n}-\frac{S_{0}}{n_{0}}\right)\right] \\
= & k n U_{0}\left(\frac{n}{V}\right)^{\frac{2}{3}} \frac{V_{0}^{2 / 3}}{n_{0}^{5 / 3}} \exp \left[\frac{2}{3 R}\left(\frac{S}{n}-\frac{S_{0}}{n_{0}}\right)\right] \\
= & k U(S, V, n) .
\end{aligned}
$$

This means that the internal energy function is a homogeneous function of degree one with respect to all extensive variables (see "Appendix 2", [6]), i.e., the following equation must be valid as a mathematical consequence (see Euler's theorem for homogeneous functions in “Appendix 3", [5, 6]):

$$
\begin{aligned}
U(S, V, n) & =\left(\frac{\partial U}{\partial S}\right)_{V, n} S+\left(\frac{\partial U}{\partial V}\right)_{S, n} V+\left(\frac{\partial U}{\partial n}\right)_{S, V} n \\
& =T S-p V+\mu n .
\end{aligned}
$$

In Eq. (6) $T$ is the temperature, $p$ is the pressure and $\mu$ is called the chemical potential.

On the other hand:

$$
\begin{aligned}
\mathrm{d} U & =\left(\frac{\partial U}{\partial S}\right)_{V, n} \mathrm{~d} S+\left(\frac{\partial U}{\partial V}\right)_{S, n} \mathrm{~d} V+\left(\frac{\partial U}{\partial n}\right)_{S, V} \mathrm{~d} n \\
& =T \mathrm{~d} S-p \mathrm{~d} V+\mu \mathrm{d} n .
\end{aligned}
$$

The partial derivatives of $U$ are:

$$
\begin{aligned}
\left(\frac{\partial U}{\partial V}\right)_{S, n}= & -\frac{2}{3} U_{0}\left(\frac{n}{V}\right)^{\frac{5}{3}} \frac{V_{0}^{2 / 3}}{n_{0}^{5 / 3}} \exp \left[\frac{2}{3 R}\left(\frac{S}{n}-\frac{S_{0}}{n_{0}}\right)\right] \\
= & -p(S, V, n) \\
\left(\frac{\partial U}{\partial S}\right)_{V, n}= & \frac{2}{3 R} U_{0}\left(\frac{n}{V}\right)^{\frac{2}{3}} \frac{V_{0}^{2 / 3}}{n_{0}^{5 / 3}} \exp \left[\frac{2}{3 R}\left(\frac{S}{n}-\frac{S_{0}}{n_{0}}\right)\right] \\
= & T(S, V, n) \\
\left(\frac{\partial U}{\partial n}\right)_{S, V}= & {\left[\frac{5}{3}\left(\frac{n}{V}\right)^{\frac{2}{3}}-\frac{2}{3 R}\left(\frac{S}{n}\right)\left(\frac{n}{V}\right)^{\frac{2}{3}}\right] n_{0}^{-5 / 3} U_{0} V_{0}^{2 / 3} } \\
& \times \exp \left[\frac{2}{3 R}\left(\frac{S}{n}-\frac{S_{0}}{n_{0}}\right)\right] \\
= & \left(\frac{n}{V}\right)^{\frac{2}{3}}\left[\frac{5}{3}-\frac{2}{3 R}\left(\frac{S}{n}\right)\right] n_{0}^{-5 / 3} U_{0} V_{0}^{2 / 3} \\
& \times \exp \left[\frac{2}{3 R}\left(\frac{S}{n}-\frac{S_{0}}{n_{0}}\right)\right]=\mu(S, V, n) .
\end{aligned}
$$

It can be seen that $p(S, V, n), T(S, V, n)$, and $\mu(S, V, n)$ are homogeneous functions of degree 0 , since: 


$$
\begin{aligned}
p(k S, k V, k n)= & \frac{2}{3} U_{0}\left(\frac{k n}{k V}\right)^{\frac{5}{3}} \frac{V_{0}^{2 / 3}}{n_{0}^{5 / 3}} \exp \left[\frac{2}{3 R}\left(\frac{k S}{k n}-\frac{S_{0}}{n_{0}}\right)\right] \\
= & p(S, V, n) \\
T(k S, k V, k n)= & \frac{2}{3 R} U_{0}\left(\frac{k n}{k V}\right)^{\frac{2}{3}} \frac{V_{0}^{2 / 3}}{n_{0}^{5 / 3}} \exp \left[\frac{2}{3 R}\left(\frac{k S}{k n}-\frac{S_{0}}{n_{0}}\right)\right] \\
= & T(S, V, n) \\
\mu(k S, k V, k n)= & \left(\frac{k n}{k V}\right)^{\frac{2}{3}}\left[\frac{5}{3}-\frac{2}{3 R}\left(\frac{k S}{k n}\right)\right] n_{0}^{-5 / 3} U_{0} V_{0}^{2 / 3} \\
& \times \exp \left[\frac{2}{3 R}\left(\frac{k S}{k n}-\frac{S_{0}}{n_{0}}\right)\right]=\mu(S, V, n) .
\end{aligned}
$$

Using Eqs. (6), (8), (9) and (10) it can be shown explicitly that $U$ is a homogeneous function of degree 1 :

$$
\begin{aligned}
U(S, V, n)=\left(\frac{\partial U}{\partial S}\right)_{V, n} S+\left(\frac{\partial U}{\partial V}\right)_{S, n} V+\left(\frac{\partial U}{\partial n}\right)_{S, V} n \\
=\frac{2}{3 R} U_{0}\left(\frac{n}{V}\right)^{\frac{2}{3}} \frac{V_{0}^{2 / 3}}{n_{0}^{5 / 3}} \exp \left[\frac{2}{3 R}\left(\frac{S}{n}-\frac{S_{0}}{n_{0}}\right)\right] S \\
\quad-\frac{2}{3} U_{0}\left(\frac{n}{V}\right)^{\frac{5}{3}} \frac{V_{0}^{2 / 3}}{n_{0}^{5 / 3}} \exp \left[\frac{2}{3 R}\left(\frac{S}{n}-\frac{S_{0}}{n_{0}}\right)\right] V \\
+\left[\frac{5}{3}\left(\frac{n}{V}\right)^{\frac{2}{3}}-\frac{2}{3 R}\left(\frac{S}{n}\right)\left(\frac{n}{V}\right)^{\frac{2}{3}}\right] U_{0} \frac{V_{0}^{2 / 3}}{n_{0}^{5 / 3}} \exp \left[\frac{2}{3 R}\left(\frac{S}{n}-\frac{S_{0}}{n_{0}}\right)\right] n \\
=U_{0}\left(\frac{V_{0}}{V}\right)^{\frac{2}{3}}\left(\frac{n}{n_{0}}\right)^{\frac{5}{3}} \exp \left[\frac{2}{3 R}\left(\frac{S}{n}-\frac{S_{0}}{n_{0}}\right)\right] \\
=n U_{0}\left(\frac{n}{V}\right)^{\frac{2}{3}} \frac{V_{0}^{2 / 3}}{n_{0}^{5 / 3}} \exp \left[\frac{2}{3 R}\left(\frac{S}{n}-\frac{S_{0}}{n_{0}}\right)\right] .
\end{aligned}
$$

Equation (7) can also be written in the explicit form as:

$$
\begin{aligned}
\mathrm{d} U= & \left(\frac{\partial U}{\partial S}\right)_{V, n} \mathrm{~d} S+\left(\frac{\partial U}{\partial V}\right)_{S, n} \mathrm{~d} V+\left(\frac{\partial U}{\partial n}\right)_{S, V} \\
\mathrm{~d} n= & T \mathrm{~d} S-p \mathrm{~d} V+\mu \mathrm{d} n \\
= & \frac{2}{3 R} U_{0}\left(\frac{n}{V}\right)^{\frac{2}{3}} \frac{V_{0}^{2 / 3}}{n_{0}^{5 / 3}} \exp \left[\frac{2}{3 R}\left(\frac{S}{n}-\frac{S_{0}}{n_{0}}\right)\right] \mathrm{d} S \\
& -\frac{2}{3} U_{0}\left(\frac{n}{V}\right)^{\frac{5}{3}} \frac{V_{0}^{2 / 3}}{n_{0}^{5 / 3}} \exp \left[\frac{2}{3 R}\left(\frac{S}{n}-\frac{S_{0}}{n_{0}}\right)\right] \mathrm{d} V \\
& +\left[\frac{5}{3}\left(\frac{n}{V}\right)^{\frac{2}{3}}-\frac{2}{3 R}\left(\frac{S}{n}\right)\left(\frac{n}{V}\right)^{\frac{2}{3}}\right] n_{0}^{-5 / 3} U_{0} V_{0}^{2 / 3} \\
& \times \exp \left[\frac{2}{3 R}\left(\frac{S}{n}-\frac{S_{0}}{n_{0}}\right)\right] \mathrm{d} n
\end{aligned}
$$

Equations (8)-(10) enable one to construct the equations of state of the ideal gas, that is:

$$
\begin{aligned}
p \cdot V & =\frac{2}{3} U_{0}\left(\frac{n}{V}\right)^{\frac{5}{3}} \frac{V_{0}^{2 / 3}}{n_{0}^{5 / 3}} \exp \left[\frac{2}{3 R}\left(\frac{S}{n}-\frac{S_{0}}{n_{0}}\right)\right] \cdot V \\
& =\frac{2}{3} U_{0}\left(\frac{n}{n_{0}}\right)^{\frac{5}{3}}\left(\frac{V_{0}}{V}\right)^{\frac{2}{3}} \exp \left[\frac{2}{3 R}\left(\frac{S}{n}-\frac{S_{0}}{n_{0}}\right)\right]
\end{aligned}
$$

on the other hand

$n \cdot R \cdot T=n R \frac{2}{3 R} U_{0}\left(\frac{n}{V}\right)^{\frac{2}{3}} \frac{V_{0}^{2 / 3}}{n_{0}^{5 / 3}} \exp \left[\frac{2}{3 R}\left(\frac{S}{n}-\frac{S_{0}}{n_{0}}\right)\right]$,

consequently,

$p \cdot V=n \cdot R \cdot T$.

By comparing Eqs. (4) and (9) we can clearly see that: $U=\frac{3}{2} n \cdot R \cdot T$.

Similarly, in the reference state $p_{0}=\frac{2}{3} \frac{U_{0}}{V_{0}}, T_{0}=\frac{2}{3 R} \frac{U_{0}}{n_{0}}$, therefore

$p_{0} \cdot V_{0}=n_{0} \cdot R \cdot T_{0}$.

and

$U_{0}=\frac{3}{2} n_{0} \cdot R \cdot T_{0}$

\section{Legendre transforms of the internal energy function}

We consider now the Legendre transforms of $U(S, V, n)$ with respect to some extensive variables. These new functions are called thermodynamic potential functions (or thermodynamic potentials): (a) the "free energy function", "Helmholtz free energy function" or "Helmholtz potential", $F$, is the Legendre transform of $U$ with respect to $S$, it is defined as:

$F=U-T S$

(b) the enthalpy function, $H$, is the Legendre transform of $U$ with respect to $V$, it is similarly defined as:

$H=U+p V$

(c) the "Gibbs free energy function", "free enthalpy function", or "Gibbs potential", G, defined as the Legendre transform of the internal energy $U$ with respect to the entropy $S$ and the volume $V$ :

$G=U-T S+p V$ 
(d) the "grand potential function", "Landau free energy function", "Kramers energy function" or "Landau potential", $\Phi$, defined as the Legendre transform of the internal energy $U$ function with respect to the entropy $S$ and the chemical amount $n$ :

$\Phi=U-T S-\mu n$

\section{The Helmholtz free energy function of an ideal monatomic gas}

The Helmholtz potential or the Helmholtz free energy, is the partial Legendre transform (see [5, 6], and "Appendix 4") of $U$ that replaces the entropy by the temperature as the independent variable. The internationally adopted symbol for the Helmholtz potential is $F$. The natural variables of the Helmholtz potential of the ideal gas are $T, V$, and $n$. That is, the functional relation $F(T, V, n)$ constitutes a fundamental relation.

The Legendre transformation of $U$

$F=U-T S$

(see Eq. 16a) can be carried out as follows: The $T(S, V, n)$ function can be inverted, i.e., from Eq. (9):

$S=S(T, V, n)=n \frac{3 R}{2} \ln \left[\frac{3 R T}{2 U_{0}}\left(\frac{n}{V}\right)^{-\frac{2}{3}} \frac{V_{0}^{-2 / 3}}{n_{0}^{-5 / 3}}\right]+n \frac{S_{0}}{n_{0}}$

By using Eqs. (4) and (16a), the Legendre transform of $U$ with respect to $S$ can be written as:

$$
\begin{aligned}
& F(T, V, n)=U-T S=n U_{0}\left(\frac{n}{V}\right)^{\frac{2}{3}} \frac{V_{0}^{2 / 3}}{n_{0}^{5 / 3}} \exp \left[\frac{2}{3 R}\left(\frac{S}{n}-\frac{S_{0}}{n_{0}}\right)\right] \\
& -T S=n U_{0}\left(\frac{n}{V}\right)^{\frac{2}{3}} \frac{V_{0}^{2 / 3}}{n_{0}^{5 / 3}} \\
& \times \exp \left[\frac{2}{3 R}\left(\frac{n \frac{3 R}{2} \ln \left[\frac{3 R T}{2 U_{0}}\left(\frac{n}{V}\right)^{-\frac{2}{3} \frac{V_{0}^{-2 / 3}}{n_{0}^{-5 / 3}}}\right]+n \frac{S_{0}}{n_{0}}}{n}-\frac{S_{0}}{n_{0}}\right)\right] \\
& -T\left(n \frac{3 R}{2} \ln \left[\frac{3 R T}{2 U_{0}}\left(\frac{n}{V}\right)^{-\frac{2}{3}} \frac{V_{0}^{-2 / 3}}{n_{0}^{-5 / 3}}\right]+n \frac{S_{0}}{n_{0}}\right)
\end{aligned}
$$

Thus

$F(T, V, n)=\frac{3}{2} n R T\left(1-\frac{2 S_{0}}{3 R n_{0}}-\ln \left[\frac{3 R T}{2 U_{0}}\left(\frac{V}{n}\right)^{\frac{2}{3}} \frac{n_{0}^{5 / 3}}{V_{0}^{2 / 3}}\right]\right)$

or by eliminating $U_{0}$ in Eq. (18) with the help of Eqs. (14a) and (15a):

$F(T, V, n)=\frac{3}{2} n R T\left(1-\frac{2 S_{0}}{3 R n_{0}}-\ln \left[\frac{T}{T_{0}}\left(\frac{V}{V_{0}}\right)^{\frac{2}{3}}\left(\frac{n_{0}}{n}\right)^{\frac{2}{3}}\right]\right)$.
It can be clearly seen that at constant temperature $F(T, V, n)$ is a homogeneous function of degree one (see "Appendix 2") in the variables $V$ and $n$, since

$$
\begin{aligned}
F(T, k V, k n) & =\frac{3}{2} k n R T\left(1-\frac{2 S_{0}}{3 R n_{0}}-\ln \left[\frac{3 R T}{2 U_{0}}\left(\frac{k V}{k n}\right)^{\frac{2}{3}} \frac{n_{0}^{5 / 3}}{V_{0}^{2 / 3}}\right]\right) \\
& =k F(T, V, n)
\end{aligned}
$$

More precisely: the function $F(T, V, n)$ is partly homogeneous of degree one in terms of the variables $V$ and $n$ ("Appendix 2").

We can note here that the chemical potential:

$$
\begin{aligned}
\mu(T, V, n) & =\left(\frac{\partial F}{\partial n}\right)_{T, V} \\
& =\frac{5}{2} R T-\frac{T S_{0}}{n_{0}}-\frac{3}{2} R T \ln \left[\frac{3 R T}{2 U_{0}}\left(\frac{V}{n}\right)^{\frac{2}{3}} \frac{n_{0}^{5 / 3}}{V_{0}^{2 / 3}}\right]
\end{aligned}
$$

can also be expressed as:

$\mu(T, V, n)=\left(\frac{\partial F}{\partial n}\right)_{T, V}=\frac{F}{n}+R T$.

\section{The enthalpy function of an ideal monatomic gas}

The enthalpy function is the partial Legendre transform (see $[5,6]$ and "Appendix 4") of $U$ that replaces the volume by the pressure as the independent variable. The internationally adopted symbol for enthalpy is $H$. The natural variables of the enthalpy are $S, p$, and $n$, that is, $H=H(S, p, n)$. The Legendre transformation of $U$.

$H=U+p V$

(see Eq. 16b) can be carried out as follows:

From Eq. (8):

$$
\begin{aligned}
V & =V(S, p, n) \\
& =\left(\frac{2}{3}\right)^{3 / 5} U_{0}^{3 / 5} p^{-3 / 5} \frac{n}{n_{0}} V_{0}^{2 / 5} \cdot \exp \left[\frac{2}{3 R}\left(\frac{S}{n}-\frac{S_{0}}{n_{0}}\right)\right]
\end{aligned}
$$

By using Eqs. (4) and (19), the Legendre transform of $U$ with respect to $V$ can be written as:

$$
\begin{aligned}
H(S, p, n)= & n^{5 / 3} U_{0} \frac{V_{0}^{2 / 3}}{n_{0}^{5 / 3}} \exp \left[\frac{2}{3 R}\left(\frac{S}{n}-\frac{S_{0}}{n_{0}}\right)\right] \\
& \times\left(\frac{2}{3}\right)^{-2 / 5} U_{0}^{-2 / 5} p^{2 / 5}\left(\frac{n}{n_{0}}\right)^{-2 / 3} V_{0}^{-4 / 15} \\
& \times \exp \left[-\frac{4}{15 R}\left(\frac{S}{n}-\frac{S_{0}}{n_{0}}\right)\right]+p \cdot\left(\frac{2}{3}\right)^{3 / 5} \\
& \times U_{0}^{3 / 5} p^{-3 / 5} \frac{n}{n_{0}} V_{0}^{2 / 5} \cdot \exp \left[\frac{2}{5 R}\left(\frac{S}{n}-\frac{S_{0}}{n_{0}}\right)\right]
\end{aligned}
$$


Thus the enthalpy function the ideal monatomic gas can be given in the explicit form as.

$$
\begin{aligned}
H(S, p, n)= & \frac{5}{2^{2 / 5} \cdot 3^{3 / 5}} \frac{n}{n_{0}} U_{0}^{3 / 5} p^{2 / 5} V_{0}^{2 / 5} \\
& \times \exp \left[\frac{2}{5 R}\left(\frac{S}{n}-\frac{S_{0}}{n_{0}}\right)\right] .
\end{aligned}
$$

On the other hand:

$$
\begin{aligned}
T(S, p, n) & =\left(\frac{\partial H}{\partial S}\right)_{p, n} \\
& =\frac{(2 / 3)^{3 / 5}}{n_{0} R} U_{0}^{3 / 5} p^{2 / 5} V_{0}^{2 / 5} \cdot \exp \left[\frac{2}{5 R}\left(\frac{S}{n}-\frac{S_{0}}{n_{0}}\right)\right] .
\end{aligned}
$$

By comparing Eqs. (20) and (21) we get

$H(S, p, n)=\frac{5}{2} n R T$.

It can be noted that at constant pressure $H(S, p, n)$ is a partly homogeneous function, i.e., a homogeneous function of degree one (see "Appendix 2") in the variables $V$ and $n$, since at constant $p$

$$
\begin{aligned}
H(k S, p, k n)= & \frac{5}{2^{2 / 5} \cdot 3^{3 / 5}} \frac{k n}{n_{0}} U_{0}^{3 / 5} p^{2 / 5} V_{0}^{2 / 5} \\
& \times \exp \left[\frac{2}{5 R}\left(\frac{k S}{k n}-\frac{S_{0}}{n_{0}}\right)\right] \\
= & k H(S, p, n)
\end{aligned}
$$

\section{The Gibbs free energy of an ideal monatomic gas}

The "Gibbs free energy", or Gibbs potential, is the (double) Legendre transform of the internal energy $U$ with respect to the entropy $S$ and the volume $V$ (see Eq. 16c) $G=U-T S+p V$.

From Eqs. (8) and (9) we get:

$V(T, p, n)=\frac{n R T}{p}$

and

$S(T, p, n)=\frac{3}{2} n R \ln \left[\frac{3}{2 U_{0}} \cdot \frac{\left(n_{0} R T\right)^{5 / 3}}{\left(p V_{0}\right)^{2 / 3}}\right]+\frac{n S_{0}}{n_{0}}$.

Thus, by taking into account that $V=V(T, p, n)$ and $S=S(T, p, n)$ :

$$
\begin{aligned}
G(T, p, n)= & n U_{0}\left(\frac{n}{V}\right)^{\frac{2}{3}} \frac{V_{0}^{2 / 3}}{n_{0}^{5 / 3}} \exp \left[\frac{2}{3 R}\left(\frac{S}{n}-\frac{S_{0}}{n_{0}}\right)\right] \\
& -T\left(\frac{3}{2} n R \ln \left[\frac{3}{2 U_{0}} \cdot \frac{\left(n_{0} R T\right)^{5 / 3}}{\left(p V_{0}\right)^{2 / 3}}\right]+\frac{n S_{0}}{n_{0}}\right)+p \frac{n R T}{p} \\
= & \frac{5}{2} n R T\left(1-\frac{2 S_{0}}{5 n_{0} R}-\frac{3}{5} \ln \left[\frac{3\left(n_{0} R T\right)^{5 / 3}}{2} p_{0}^{-2 / 3}\right]\right)
\end{aligned}
$$

that is

$$
G(T, p, n)=\frac{5}{2} n R T\left(1-\frac{2 S_{0}}{5 n_{0} R}-\frac{3}{5} \ln \left[\frac{3}{2} \frac{\left(n_{0} R T\right)^{5 / 3}}{U_{0} V_{0}^{2 / 3}} p^{-2 / 3}\right]\right)
$$

or by taking into account Eqs. (14a) and (15a):

$G(T, p, n)=\frac{5}{2} n R T\left(1-\frac{2 S_{0}}{5 n_{0} R}-\frac{3}{5} \ln \left[\left(\frac{T}{T_{0}}\right)^{5 / 3}\left(\frac{p_{0}}{p}\right)^{2 / 3}\right]\right)$.

Note, that there is apparently a small algebraic mistake in the relevant expression for $G$ given in [7, p. 64].

Alternatively, the Gibbs free energy function can be obtained as:

$G=F+p V$

or

$G=H-T S$

In the first case, by using Eqs. (18), (23) and (24) we obtain that

$$
\begin{aligned}
G(T, p, n) & =\frac{3}{2} n R T\left(1-\frac{2 S_{0}}{3 R n_{0}}-\ln \left[\frac{3 R T}{2 U_{0}}\left(\frac{V}{n}\right)^{\frac{2}{3}} \frac{n_{0}^{5 / 3}}{V_{0}^{2 / 3}}\right]\right)+p V \\
& =\frac{3}{2} n R T\left(1-\frac{2 S_{0}}{3 R n_{0}}-\ln \left[\frac{3 R T}{2 U_{0}}\left(\frac{n R T}{n p}\right)^{\frac{2}{3}} \frac{n_{0}^{5 / 3}}{V_{0}^{2 / 3}}\right]\right)+p \cdot \frac{n R T}{p} \\
& =\frac{5}{2} n R T\left(1-\frac{2 S_{0}}{5 n_{0} R}-\frac{3}{5} \ln \left[\frac{3}{2} \frac{\left(n_{0} R T\right)^{5 / 3}}{U_{0} V_{0}^{2 / 3}} p^{-2 / 3}\right]\right),
\end{aligned}
$$

and in the second case we can obtain Eq. (25) from Eqs. (20), (23) and (24).

We note here that 


$$
\begin{aligned}
\mu(T, p, n) & =\left(\frac{\partial G(T, p, n)}{\partial n}\right)_{T, p} \\
& =\frac{5}{2} R T\left(1-\frac{2 S_{0}}{5 n_{0} R}-\frac{3}{5} \ln \left[\frac{3}{2} \frac{\left(n_{0} R T\right)^{5 / 3}}{U_{0} V_{0}^{2 / 3}} p^{-2 / 3}\right]\right) \\
& =\frac{G(T, p, n)}{n}
\end{aligned}
$$

and $\mu$ is independent of $n$. The same conclusion also follows from the fact, that $G(T, p, n)$ is a homogeneous function of degree one with respect to $n$ (see "Appendix 2", especially Eq. (60); in order to be more precise: the function $G$ is partly homogeneous of degree one in terms of the variable $n$ ):

$$
\begin{aligned}
G(T, p, k n)= & \frac{5}{2^{2 / 5} \cdot 3^{3 / 5}} \frac{k n}{n_{0}} U_{0}^{3 / 5} p^{2 / 5} V_{0}^{2 / 5} \\
& \times \exp \left[\frac{2}{5 R}\left(\frac{k S}{k n}-\frac{S_{0}}{n_{0}}\right)\right]-T S \\
= & \frac{5}{2} k n R T\left(1-\frac{2 S_{0}}{5 n_{0} R}-\frac{3}{5} \ln \left[\frac{3}{2} \frac{\left(n_{0} R T\right)^{5 / 3}}{U_{0} V_{0}^{2 / 3}} p^{-2 / 3}\right]\right) \\
= & k G(T, p, n) .
\end{aligned}
$$

\section{The grand potential function of an ideal monatomic gas}

The "grand potential function" is the (double) Legendre transform of the internal energy $U$ with respect to the entropy $S$ and the volume chemical amount $n$ (see Eq. 16d) $\Phi=U-T S-\mu n$.

From Eqs. (9) and (10) we get:

$n(T, V, \mu)=\frac{V}{V_{0}}\left(\frac{3}{2}\right)^{3 / 2} n_{0}\left(\frac{n_{0} R T}{U_{0}}\right)^{3 / 2} \exp \left[\frac{\mu}{R T}+\frac{S_{0}}{n_{0} R}-\frac{5}{2}\right]$

and

$$
\begin{aligned}
S(T, V, \mu)= & \frac{V}{V_{0}}\left(\frac{3}{2}\right)^{3 / 2} n_{0}\left(\frac{n_{0} R T}{U_{0}}\right)^{3 / 2}\left(\frac{5}{2} R-\frac{\mu}{T}\right) \\
& \times \exp \left[\frac{\mu}{R T}+\frac{S_{0}}{n_{0} R}-\frac{5}{2}\right] .
\end{aligned}
$$

The result of the double Legendre transformation of $U(S, V, n)$ with respect to $S$ and $n$ is:

$\Phi(T, V, \mu)=-\frac{V}{V_{0}}\left(\frac{3}{2}\right)^{3 / 2} \frac{\left(n_{0} R T\right)^{5 / 2}}{U_{0}^{3 / 2}} \cdot \exp \left[\frac{\mu}{R T}+\frac{S_{0}}{n_{0} R}-\frac{5}{2}\right]$

We can easily prove that $\Phi(T, V, \mu)$ is a homogeneous function of degree one with respect to $V$.
On the other hand, by introducing $n(T, V, \mu)$ and $S(T, V, \mu)$ [see Eqs. (27) and (28)] into Eq. (8), we find that the pressure $p$ can be expressed as follows:

$p(T, V, \mu)=\left(\frac{3}{2}\right)^{3 / 2} \frac{\left(n_{0} R T\right)^{5 / 2}}{V_{0} U_{0}^{3 / 2}} \cdot \exp \left[\frac{\mu}{R T}+\frac{S_{0}}{n_{0} R}-\frac{5}{2}\right]$

From Eqs. (29) and (30) we can also see that $p$ is independent of $V$, and

$p(T, V, \mu)=-\left(\frac{\partial \Phi(T, V, \mu)}{\partial V}\right)_{T, p}=-\frac{\Phi(T, V, \mu)}{V}$.

We note here that functions like $\Phi(T, V, \mu)$ are sometimes called "mechanical work functions". In statistical mechanics, it is often referred to as "grand canonical potential".

\section{Other Legendre transforms of the $U(S, V, n)$ function}

Other possible Legendre transforms of the internal energy function $U(S, V, n)$, which are used only infrequently and which consequently are unnamed, are:

$U(S, V, \mu)=U-\mu n$

and

$U(S, p, \mu)=U-T S-\mu n$.

It is to be remarked here, however, that in these cases the equations for the inversions appear to involve the variables to be solved for in an essentially non-algebraic way.

The "complete" Legendre transform of $U(S, V, n)$ is $U(T, p, \mu)$. The fact that $U(S, V, n)$ is a homogeneous function of degree one with respect to all of its arguments causes this latter function to vanish identically. For:

$U(T, p, \mu)=U-T S+p V-\mu n$

which, by the Euler relation (see "Appendix 3"), is identically zero, i.e.,

$U(T, p, \mu) \equiv 0$.

It should be stressed, however, that the above identity is valid only for homogeneous functions of degree one. For example, with $f_{x}=\frac{\partial f}{\partial x}=e^{y}, f_{y}=\frac{\partial f}{\partial y}=x e^{y}, x=\frac{f_{y}}{f_{x}}$, and $y=\ln f_{x}$, respectively, the (double) Legendre transform of the function.

$f(x, y)=x \cdot e^{y}$

can be expressed as: 


$$
\begin{aligned}
g\left(f_{x}, f_{y}\right) & =\frac{f_{y}}{f_{x}} \cdot f_{x}-\frac{f_{y}}{f_{x}} \cdot f_{x}-f_{y} \\
\ln f_{x} & =-f_{y} \ln f_{x} \neq 0 .
\end{aligned}
$$

\section{Some notes concerning the derivatives of the thermodynamic potential functions}

The temperature, pressure, and the chemical potential are partial derivatives of functions of $S, V$, and $n$ and consequently are also functions of $S, V$ and $n$. We thus have a set of functional relationships (see Eqs. 8-10):

$p=p(S, V, n)$

$T=T(S, V, n)$

$\mu=\mu(S, V, n)$.

The expressions for $p, T$ and $\mu$ can be obtained from the other thermodynamic potential functions by partial derivation.

For example, the chemical potential functions can be derived from the thermodynamic potential functions by taking partial derivatives of the functions with respect to the chemical amount $n$, and can be written as:

$$
\begin{aligned}
\mu(S, V, n)= & \left(\frac{\partial U}{\partial n}\right)_{S, V}=\left(\frac{n}{V}\right)^{\frac{2}{3}}\left[\frac{5}{3}-\frac{2}{3 R}\left(\frac{S}{n}\right)\right] n_{0}^{-5 / 3} U_{0} V_{0}^{2 / 3} \\
& \times \exp \left[\frac{2}{3 R}\left(\frac{S}{n}-\frac{S_{0}}{n_{0}}\right)\right] \\
\mu(T, V, n)= & \left(\frac{\partial F}{\partial n}\right)_{T, V} \\
= & \frac{5}{2} R T-\frac{T S_{0}}{n_{0}}-\frac{3}{2} R T \ln \left[\frac{3 R T}{2 U_{0}}\left(\frac{V}{n}\right)^{\frac{2}{3}} \frac{n_{0}^{5 / 3}}{V_{0}^{2 / 3}}\right]
\end{aligned}
$$

$$
\begin{aligned}
\mu(S, p, n)= & \left(\frac{\partial H}{\partial n}\right)_{S, p} \\
= & \frac{5}{2^{2 / 5} \cdot 3^{3 / 5}}\left[1-\frac{2}{5 R}\left(\frac{S}{n}\right)\right] \frac{U_{0}^{3 / 5}}{n_{0}} p^{2 / 5} V_{0}^{2 / 5} \\
& \times \exp \left[\frac{2}{5 R}\left(\frac{S}{n}-\frac{S_{0}}{n_{0}}\right)\right], \\
\mu(T, p, n)= & \left(\frac{\partial G}{\partial n}\right)_{T, p} \\
= & \frac{5}{2} R T-\frac{T S_{0}}{n_{0}}-\frac{5}{3} R T \ln \left[\frac{3}{2} \frac{\left(n_{0} R T\right)^{5 / 3}}{U_{0} V_{0}^{2 / 3}} p^{-2 / 3}\right],
\end{aligned}
$$

that is the chemical potential functions are defined by "different equations".
On the other hand, it follows from the properties of fundamental relations that for a given set of the extensive variables $\left(S_{1}, V_{1}, n_{1}\right)$, i.e., in a given state, the value of $\mu$ is unambiguously determined (together with the values of the other intensive variables). Consequently

$$
\begin{aligned}
\mu\left(S_{1}, V_{1}, n_{1}\right) & =\mu\left(T_{1}, V_{1}, n_{1}\right)=\mu\left(S_{1}, p_{1}, n_{1}\right) \\
& =\mu\left(T_{1}, p_{1}, n_{1}\right) .
\end{aligned}
$$

This can be easily proved by substituting the relevant expressions of $T$ and $p$ into Eqs. (39)-(41). (It is obvious that equivalent relationships exist for the relevant $T$ and $p$ functions as well).

The above example show that there is a difference in meaning between a function $f(\boldsymbol{x})$ and the value of the function at the point $\boldsymbol{x}$, that is the "function" and the "function value" are two distinct concepts. In classical mathematics there has been no need to introduce separate symbols for those concepts. In certain branches of mathematics and in physics, however, this distinction becomes absolutely necessary [8]. A rigorous distinction may seem subtle in thermodynamics, but it is quite fundamental, because confusing the two concepts can lead to a number of errors and misinterpretations [9].

\section{Concluding remarks}

The fundamental functions in thermodynamics $(U(S, V$, $\left.n_{1}, \ldots, n_{\mathrm{m}}, \ldots\right)$ or $\left.S\left(U, V, n_{1}, \ldots, n_{\mathrm{m}}, \ldots\right)\right)$ are probably the most fundamental but not the most practical relations of thermodynamics. In many situations, the variables used in these expressions are not the ones controlled or measured in practice. For instance, we cannot measure the entropy (or the internal energy) directly, so that it must be calculated in terms of "measurable" ("controllable" or "laboratory") variables, which are, e.g., temperature, pressure, volume, chemical amount, density, etc. It is well known that with the help of Legendre transformations, fundamental equations can be transformed with conservation of their fundamental nature, i.e., the property that they contain all information about the thermodynamic properties of the system. Nevertheless, some familiarity with basic mathematical concepts is necessary for the calculations.

The internal energy function of a simple ideal gas is probably the simplest example of a fundamental function (fundamental relation) which can be given in an explicit form. Due to its properties, this function can be effectively used to study and demonstrate the application of important mathematical concepts (e.g., the theory of homogeneous functions and partly homogeneous functions, Euler's theorem, and the Legendre transformation) to real thermodynamic problems. 
Acknowledgments Financial support from the Hungarian Scientific Research Fund (Grant No. K 109036) is gratefully acknowledged.

\section{Appendix 1}

The derivation of the entropy fundamental equation of a simple ideal gas

A "simple" ideal gas is characterized by the equations:

$U=c n R T$,

and

$p V=n R T$,

where $c$ is a constant (e.g., for a "monatomic ideal gas" $c=3 / 2$ ), $n$ is the (chemical) amount of the substance, $p$ is the pressure, $V$ is the volume, $T$ is the temperature, and $R$ is the "universal" gas constant, respectively.

We can rewrite the above equations as:

$\frac{1}{T}=c R \frac{n}{U}=\frac{c R}{u}$,

and

$\frac{p}{T}=R \frac{n}{V}=\frac{R}{v}$.

The differential form of the entropy based fundamental equation is

$\mathrm{d} S=\frac{1}{T} \mathrm{~d} U+\frac{p}{T} \mathrm{~d} V-\sum_{i} \frac{\mu_{i}}{T} \mathrm{~d} n_{i}$,

and the integrated form of Eq. (47) can be written as (see Euler's theorem for homogeneous functions, Appendix 3)

$S=\frac{1}{T} U+\frac{p}{T} V-\sum_{i} \frac{\mu_{i}}{T} n_{i}$.

The entropy-based Gibbs-Duhem equation follows immediately from Eq. (48):

$U \mathrm{~d} \frac{1}{T}+V \mathrm{~d} \frac{p}{T}-\sum_{i} n_{i} \mathrm{~d} \frac{\mu_{i}}{T}=0$,

and for a one-component system:

$U \mathrm{~d} \frac{1}{T}+V \mathrm{~d} \frac{\mathrm{p}}{T}-n \mathrm{~d} \frac{\mu}{T}=0$.

Dividing Eq. (50) by the amount of substance $n$ and rearranging, we get

$\mathrm{d} \frac{\mu}{T}=u \mathrm{~d} \frac{1}{T}+v \mathrm{~d} \frac{p}{T}$.
Since $\quad \mathrm{d} \frac{1}{T}=\left(\frac{\mathrm{d} \frac{1}{T}}{\mathrm{~d} u}\right) \mathrm{d} u \quad$ and $\quad \mathrm{d} \frac{p}{T}=\left(\frac{\mathrm{d} \frac{p}{T}}{\mathrm{~d} v}\right) \mathrm{d} v, \quad$ with Eqs. (45), (46) we obtain:

$$
\begin{aligned}
\mathrm{d} \frac{\mu}{T} & =u\left(-\frac{c R}{u^{2}}\right) \mathrm{d} u+v\left(-\frac{R}{v^{2}}\right) \mathrm{d} v \\
& =-c R \frac{\mathrm{d} u}{u}-R \frac{\mathrm{d} v}{v}
\end{aligned}
$$

The first term on the right hand side of (52) is a function of only $u$, the second term is a function of only $v$, therefore we can integrate the first term only with respect to $u$ and the second one only with respect to $v$ :

$\int_{(\mu / T)_{0}}^{\mu / T} \mathrm{~d} \frac{\mu}{T}=-c R \int_{u_{0}}^{u} \frac{\mathrm{d} u}{u}-R \int_{v_{0}}^{v} \frac{\mathrm{d} v}{v}$,

that is

$\frac{\mu}{T}=-c R \ln \frac{u}{u_{0}}-R \ln \frac{v}{v_{0}}+\left(\frac{\mu}{T}\right)_{0}$.

In Eqs. (53) and (54) $u_{0}$ and $v_{0}$ are the parameters of a fixed reference state, and $(\mu / T)_{0}$ arises as an undetermined constant of integration.

By substituting $1 / T=c R / u, p / T=R / v$, and $\mu / T$ from Eqs. (45), (46) and (53), respectively, into Eq. (48) we have:

$$
\begin{aligned}
S= & \frac{1}{T} U+\frac{p}{T} V-\frac{\mu}{T} n \\
= & \frac{c R}{u} U+\frac{R}{v} V+c n R \ln \frac{u}{u_{0}}+n R \ln \frac{v}{v_{0}}-n\left(\frac{\mu}{T}\right)_{0} . \\
& \text { Since } \quad U / u=V / v=n, \frac{u}{u_{0}}=\frac{U}{n} \frac{n_{0}}{U_{0}}, \quad \text { and } \quad \frac{v}{v_{0}}=\frac{V}{n} \frac{n_{0}}{V_{0}}
\end{aligned}
$$

Eq. (55) can be write in terms of extensive variables:

$$
\begin{aligned}
S= & n c R+n R+c n R \ln \frac{n_{0} U}{U_{0} n}+n R \ln \frac{n_{0} V}{V_{0} n} \\
& -n\left(\frac{\mu}{T}\right)_{0}=(c+1) n R-n\left(\frac{\mu}{T}\right)_{0} \\
& +n R \ln \left[\left(\frac{U}{U_{0}}\right)^{c} \frac{V}{V_{0}}\left(\frac{n_{0}}{n}\right)^{c+1}\right],
\end{aligned}
$$

or by introducing

$S_{0}=S_{0}\left(U_{0}, V_{0}, n_{0}\right)=n_{0}\left[(c+1) R-\left(\frac{\mu}{T}\right)_{0}\right]$,

i.e., the entropy in the reference state, we arrive at the entropy fundamental equation of the simple ideal gas

$S=\frac{n}{n_{0}} S_{0}+n R \ln \left[\left(\frac{U}{U_{0}}\right)^{c}\left(\frac{V}{V_{0}}\right)\left(\frac{n_{0}}{n}\right)^{c+1}\right]$

or 
$S=n s_{0}+n R \ln \left[\left(\frac{U}{U_{0}}\right)^{c}\left(\frac{V}{V_{0}}\right)\left(\frac{n}{n_{0}}\right)^{-(c+1)}\right]$.

\section{Appendix 2}

\section{Homogeneous functions, partly homogeneous functions}

\section{Definition}

A homogeneous function is a function of one or several variables that satisfies the following condition: when all independent variables of a function are simultaneously multiplied by the same (arbitrary) factor, the value of the function is multiplied by some power of this factor. That is, if:

$f\left(k x_{1}, k x_{2}, \ldots, k x_{m}\right)=k^{n} f\left(x_{1}, x_{2}, \ldots, x_{m}\right)$

for all $k>0$, then $f$ is said to be a homogeneous function of degree $n$. The degree $n$ can take on any value (positive, negative, or zero). A function $f$ is linearly homogenous if it is homogeneous of degree 1.

If for a function $f$ the equation

$f\left(k x_{1}, \ldots, k x_{m}, y_{1}, \ldots, y_{w}\right)=k^{n} f\left(x_{1}, \ldots, x_{m}, y_{1}, \ldots, y_{w}\right)$

is true then we say that this function is homogeneous of degree $n$ in the variables $x_{1}, x_{2}, \ldots, x_{m}$. Such functions are called partly (or partially) homogeneous functions $[5,6,10]$. A function $f$ is called "partly homogeneous" of degree 1 in terms of $m$ among $m+w$ variables if

$f\left(k x_{1}, \ldots, k x_{m}, y_{1}, \ldots, y_{w}\right)=k f\left(x_{1}, \ldots, x_{m}, y_{1}, \ldots, y_{w}\right)$

i.e., the function $f$ is homogeneous of degree one with respect to certain variables $\left(x_{1}, x_{2}, \ldots, x_{m}\right)$, but not homogeneous with respect to all of the variables. These functions are important as they are frequently encountered in thermodynamics. For more details see, e.g., [5, 6, 11]. Remark: It can be shown [6] that if $f\left(x_{1}, x_{2}, \ldots, x_{m}\right)$ is a homogeneous function of degree $n$, it can be represented by

$f=x_{1}^{n} g\left(\frac{x_{2}}{x_{1}}, \ldots, \frac{x_{m}}{x_{1}}\right)$

with some function $g$. Since, conversely, every $f$ function formed by means of an appropriate function $g$ of $m-1$ variables satisfies the condition of homogeneity, the expression (63) represents the totality of homogeneous functions of degree $n$.

\section{Appendix 3}

\section{Euler's theorem}

Euler's theorem states that, the differentiable function $f$ of $m$ variables is homogeneous of degree $n$ then the following identity holds

$n f\left(x_{1}, x_{2}, \ldots, x_{m}\right)=\sum_{i=1}^{m} x_{i} \frac{\partial f}{\partial x_{i}}$.

It can be shown that the converse theorem also holds, that is if the function $f$ of the real variables $x_{1}, x_{2}, \ldots, x_{m}$ satisfies the identity (64), then the function $f$ is homogeneous of degree $n$. (see Refs. [6, 12] for more details).

\section{Appendix 4}

\section{Legendre transformation}

Let $f\left(x_{1}, x_{2}, \ldots, x_{m}\right)$ an arbitrary analytic function of variables $x_{1}, x_{2}, \ldots, x_{m}$. The differential of $f$ is:

$$
\begin{aligned}
\mathrm{d} f & =\frac{\partial f}{\partial x_{1}} \mathrm{~d} x_{1}+\frac{\partial f}{\partial x_{2}} \mathrm{~d} x_{2}+\cdots+\frac{\partial f}{\partial x_{m}} \mathrm{~d} x_{m} \\
& =p_{1} \mathrm{~d} x_{1}+p_{2} \mathrm{~d} x_{2}+\cdots+p_{m} \mathrm{~d} x_{m} .
\end{aligned}
$$

Consider a new function $g$ of the variables $p_{1}$ and $x_{2}, x_{3}, \ldots$, $x_{m}$ :

$g\left(p_{1}, x_{2}, \ldots, x_{m}\right)=f\left(x_{1}\left(p_{1}\right), x_{2}, \ldots, x_{m}\right)-p_{1} x_{1}\left(p_{1}\right)$,

where $p_{1}=\frac{\partial f}{\partial x_{1}}$. A necessary condition is the existence of a one to one relation between $p_{1}$ and $x_{1}$; that is, the function $p_{1}\left(x_{1}, x_{2}, \ldots, x_{m}\right)$ can be inverted to give $x_{1}\left(p_{1}\right)$. (This means that $p_{1}$ is bijective). (Note, that the transformation (66) is often called the "negative Legendre transform", and the Legendre transform is then defined as: $g\left(p_{1}, x_{2}, \ldots, x_{m}\right)=$ $p_{1} x_{1}\left(p_{1}\right)-f\left(x_{1}\left(p_{1}\right), x_{2}, \ldots, x_{m}\right)$. For more details see Refs. $[1,2,6,13,14])$.

\section{References}

1. Callen HB (1985) Thermodynamics and an introduction to thermostatistics, 2nd edn. Wiley, New York

2. Shell MS (2015) Thermodynamics and Statistical Mechanics, Cambridge University Press, University Printing House, Cambridge

3. Keszei E (2012) Chemical Thermodynamics. Springer Science \& Business Media, Berlin Heidelberg

4. Compendium of chemical terminology gold book (2014) International Union of Pure and Applied Chemistry, Version 2.3.3, p. 707, http://goldbook.iupac.org/PDF/goldbook.pdf. Accessed 24 Feb 2014 
5. Láng GG, Barbero C (2012) Laser techniques for the study of electrode processes. Springer Verlag, Heidelberg, London, New York

6. Láng GG (2015) ChemTexts16:1-16

7. Callen HB (1986) Solutions manual for Thermodynamics and an introduction to thermostatistics, 2nd edn. Wiley, New York

8. Mikusinski J (1983) Operational calculus, vol I. PWN-Polish Scientific Publishers Warszawa, Pergamon Press, Oxford

9. Láng GG, Heusler KE (1999) J Electroanal Chem 472:168-173

10. Annamalai K, Puri IK (2002) Advanced thermodynamics engineering. CRC Press, CRC Series in Computational Mechanics and Applied Analysis, Boca Raton
11. Courant R, Hilbert D (1966) Methods of mathematical physics, Vol. II, Partial differential equations. Interscience Pubs., Wiley, New York

12. Tykodi RJ (1982) J Chem Educ 59:557-559

13. Alberty RA (2001) Pure Appl Chem 73:1349-1380

14. Arnold VI (1989) Mathematical methods of classical mechanics, 2nd edn. Springer, New York 